\title{
Differentiation Inductions Altered Telomere Length and Telomerase Activity in Human Dental Pulp- Derived Mesenchymal Stem Cell
}

\author{
Hyeon-Jeong Lee, ${ }^{1, \#}$, Ryoung-Hoon Jeon ${ }^{1, \#}$, Byung-Joon Park ${ }^{2}$, Si-Jung Jang ${ }^{1}$, Sung-Lim Lee ${ }^{1}$, \\ Gyu-Jin Rho ${ }^{1}$, Seung-Joon Kim ${ }^{2}$ and Won-Jae Lee ${ }^{2, *}$ \\ ${ }^{1}$ College of Veterinary Medicine, Gyeongsang National University, Jinju 52828, Korea \\ ${ }^{2}$ College of Veterinary Medicine, Kyungpook National University, Daegu 41566, Korea
}

Received June 13, 2019

Revised June 20, 2019

Accepted June 21, 2019

\section{*Correspondence}

Won-Jae Lee

Department of Theriogenology, College of Veterinary Medicine, Kyungpook National University, 80 Daehak-ro, Buk-gu, Daegu 41566, Korea

Tel: +82-53-950-5965

Fax: +82-53-950-5994

E-mail: iamcyshd@knu.ac.kr

ORCID

https://orcid.org/0000-0003-1462-7798

${ }^{\#}$ Both first authors equally contributed to the present study.

\begin{abstract}
Telomeres are known as a specialized region in the end of chromosomes to protect DNA destruction, but their lengths are shortened by repetition of cell division. This telomere shortening can be preserved or be elongated by telomerase and TERT expression. Although a certain condition in the cells may affect to the cellular and molecular characteristics, the effect of differentiation induction to telomere length and telomerase activity in mesenchymal stem cells (MSCs) has been less studied. Therefore, the present study aimed to uncover periodical alterations of telomere length, telomerase activity and TERT expression in the dental pulp-derived MSCs (DPMSCs) under condition of differentiation inductions into adipocytes and osteoblasts on a weekly basis up to 3 weeks. Shortening of telomere was significantly $(p<$ 0.05 ) identified from early-middle stages of both differentiations in comparison with undifferentiated DP-MSCs by non-radioactive chemiluminescent assay and QRT-PCR method. Telomere length in undifferentiated DP-MSCs was $10.5 \mathrm{~kb}$, but the late stage of differentiated DP-MSCs which can be regarded as the adult somatic cell exhibited 8.1-8.6 kb. Furthermore, the relative-quantitative telomerase repeat amplification protocol or western blotting presented significant $(p<0.05)$ decrease of telomerase activity since early stages of differentiations or TERT expression from middle stages of differentiations than undifferentiated state, respectively. Based on these results, it is supposed that shortened telomere length in differentiated DP-MSCs was remained along with prolonged differentiation durations, possibly due to weakened telomerase activity and TERT expression. We expect that the present study contributes on understanding differentiation mechanism of MSCs, and provides standardizing therapeutic strategies in clinical application of MSCs in the animal biotechnology.
\end{abstract}

Keywords: differentiation, mesenchymal stem cells, telomerase, telomere

\section{INTRODUCTION}

Telomeres are known as a specialized region of repetitive DNA sequence of TTAGGG in the end of chromo- somes to protect from DNA destruction, but their lengths are gradually shortened by repetition of cell division, which results to the cell cycle arrest and senescence (Jeon et al., 2011b). However, shortening of telomere length can 
be preserved or be elongated by synthesizing telomeres de novo of telomerase activity (Artandi and DePinho, 2010). It has been known that the telomerase activity is mainly regulated by expression of telomerase reverse transcriptase (TERT) (Gonzalo et al., 2006).

Due to self-renewal, differentiation ability, pluripotency, immunomodulatory capacity and less ethical issue, mesenchymal stem cells (MSCs) have been widely investigated for the clinical application (Lee et al., 2019). Especially, they can be easily obtained from various tissues including adipose tissue (AT-MSCs), bone marrow (BMMSCs), umbilical cord matrix (UCM-MSCs) and dental pulp (DP-MSCs), and exhibit differentiation ability toward multi-lineages with respect to adipocytes, osteoblasts, chondrocytes, myocytes, neurocytes and hepatocytes (Jeon et al., 2011b; Lee et al., 2019). It has been addressed that upregulation of telomerase activity in embryonic stem cells (ESCs) plays a pivotal role in preserving telomere length, maintaining undifferentiated status and immortality (Campisi and Yaswen, 2009). Likewise, telomere length in MSCs is also considered as a necessary factor for their self-renewal and lineage differentiation ability (Jeon et al., 2011b). However, telomerase activity in MSCs has a limited capacity after large expansion, and was weakened after chondrogenic differentiation (Parsch et al., 2004; Campisi and Yaswen, 2009).

Although a certain condition may affect to the cellular and molecular characteristics of MSCs, the effect of differentiation induction to telomere length and telomerase activity in MSCs has been less studied. Because these concerns need to be clearly addressed before clinical application of MSCs, the present study aimed to uncover periodical alterations of telomere length, telomerase activity and TERT expression in DP-MSCs under condition of differentiation inductions into adipocytes and osteoblasts on a weekly basis up to 3 weeks. We expect that the present study contributes on understanding differentiation mechanism of MSCs, and provides standardizing therapeutic strategies in clinical application of MSCs in the animal biotechnology.

\section{MATERIALS AND METHODS}

\section{Chemicals and media}

Unless stated otherwise, all chemicals and media were purchased from Sigma-Aldrich Chemical Inc. (St. Louis,
MO, USA) and Thermo Fisher Scientific (Waltham, MA, USA).

\section{MSCs isolation, cultivation and characterization}

The human dental pulp tissues $(n=4)$ during extraction of the wisdom teeth were collected from Gyeongsang National University Hospital under approved guidelines (GNUH IRB-2012-09-004) after obtaining informed consents from patients. In accordance with previous article, DP-MSCs were isolated from the dental pulp tissues, and were cultivated until passage 4 for further analysis (Lee et al., 2019). MSCs-specific molecules expression (CD44, CD90 and CD105) and negative expression of hematopoietic cell marker (CD45) were investigated in DP-MSCs in accordance with previous article (Lee et al., 2019). Then DP-MSCs were differentiated toward adipocytes and osteoblasts under the same induction condition with previous article up to 3 weeks; the Oil red O staining to identify the accumulation of intracellular lipid droplets in adipogenesis and von Kossa staining to detect calcium deposits in osteogenesis were performed (Lee et al., 2019). The experimental groups were categorized into 7 groups consisting of undifferentiated DP-MSCs (Con) and differentiated DP-MSCs until the early (1 week), middle ( 2 weeks) and late (3 weeks) stages into adipocytes (A1W, $\mathrm{A} 2 \mathrm{~W}$ and $\mathrm{A} 3 \mathrm{~W})$ or osteoblasts (O1W, O2W and O3W).

\section{Telomere length assay by southern blotting}

Telomere lengths were investigated in triplicates by non-radioactive chemiluminescent assay with the TeloTAGGG telomere restriction fragment (TRF) length assay kit (Roche, IN, USA), in accordance with manufacturer' $s$ instructions. The extraction of genomic DNA of each group was done by using the Qiagen DNeasy Blood and Tissue kit (Qiagen, CA, USA), following the manufacturer' $s$ instructions. A total of $1 \mu \mathrm{g}$ genomic DNA was digested with a mixture of HinfI and RsaI restriction enzymes at $37^{\circ} \mathrm{C}$ for $2 \mathrm{~h}$. DNA fragments were separated within $0.8 \%$ agarose gel electrophoresis at $30 \mathrm{~V} / \mathrm{cm}$ for $5 \mathrm{~h}$. After gel depurination and denaturation, southern transfer of separated DNA fragments onto positively charged nylon membrane (Roche) was done by capillary transfer method for overnight. DNA was fixed to the membrane by UV cross linking (UVP, CA, USA) under $120 \mathrm{~mJ}$. The membrane was hybridized with a digoxigenin (DIG)-labeled telomere probe at $42^{\circ} \mathrm{C}$ for $3 \mathrm{~h}$, was blocked, and was incubated 
with anti-DIG-alkaline-phosphatase (AP) solution for 30 min. The membrane was then reacted with AP substrate, and was exposed to X-ray film. Values of $\mathrm{OD}_{\mathrm{i}}$ (the chemiluminescent signal of sample i) and Li (the length of the TRF at position of sample i) from scanned films were evaluated by image J (National Institute of Health, USA), and means of TRF length were defined in accordance with the formula in manufacturer's instruction: $T R F=\Sigma\left(\mathrm{OD}_{\mathrm{i}}\right) /$ $\Sigma\left(\mathrm{OD}_{\mathrm{i}} / \mathrm{L}_{\mathrm{i}}\right)$.

\section{Telomere length assay by qRT-PCR}

Telomere length was measured in triplicates using Relative Human Telomere Length Quantification qPCR Assay Kit (ScienCell, CA, USA), in accordance with manufacturer's instruction. Genomic DNA was isolated from Con and differentiated DP-MSCs using QIAamp ${ }^{\circledR}$ DNA Mini Kit (Qiagen). Quantitative real-time PCR (qRT-PCR) was performed using Rotor Gene Q cycler (Qiagen) with RealMOD $^{\mathrm{TM}}$ Green AP $5 \times$ qPCR mix (iNtRON Biotechnology, Seongnam, Korea) supplemented with 5 ng cDNA, telomere primer and single copy reference (SCR) primer. PCR cycling program consisted of initial activation at $95^{\circ} \mathrm{C}$ for $12 \mathrm{~min}$, followed by 40 cycles of PCR at $95^{\circ} \mathrm{C}$ for $15 \mathrm{sec}$, $60^{\circ} \mathrm{C}$ for $25 \mathrm{sec}$ and $72^{\circ} \mathrm{C}$ for $25 \mathrm{sec}$. The cycle threshold values (Ct values) were analyzed by Rotor-Gene Q Series Software (Qiagen). Ct values from telomere primer of each sample were normalized against the values obtained from SCR primer.

\section{Analysis of telomerase activity}

The relative-quantitative telomerase repeat amplification protocol (RQ-TRAP) to detect telomerase activity was performed in triplicates in accordance with previous reports (Jeon et al., 2011a). The number of $1 \times 10^{5}$ cells from Con and differentiated DP-MSCs were harvested, and were immediately frozen at $-80^{\circ} \mathrm{C}$. The cells were lysed with $1 \cdot 3$-[(3-cholamidopropyl) dimethylammonio] propanesulfonic acid lysis buffer (CHAPS, Chemicon international, CA, USA) containing $100 \mathrm{U} / \mathrm{mL}$ RNase inhibitor for $30 \mathrm{~min}$ on ice. Lysates were then centrifuged for $20 \mathrm{~min}$ at $12,000 \mathrm{~g}$ at $4^{\circ} \mathrm{C}$. The mixture for RQ-TRAP consisted of $12.5 \mu \mathrm{L} 2 \times$ Rotor-Gene SYBR Green (Qiagen), $0.02 \mu$ g primer TS (5'-AAT CCG TCG GAG CAG AGT T-3'), $0.04 \mu \mathrm{g}$ primer ACX (5'-GCG CGG CTT ACC CTT ACC CTT ACC CTA ACC-3') and $5 \mathrm{mg}$ total protein from each sample. RQ-TRAP was performed using Rotor Gene
Q cycler (Qiagen) under incubation for $30 \mathrm{~min}$ at $30^{\circ} \mathrm{C}$, followed by incubation $10 \mathrm{~min}$ at $94^{\circ} \mathrm{C}$ and 40 cycles of PCR at $95^{\circ} \mathrm{C}$ for $30 \mathrm{sec}$ and $60^{\circ} \mathrm{C}$ for $90 \mathrm{sec}$. The Ct values were analyzed by Rotor-Gene Q Series Software (Qiagen). Ct values from differentiated DP-MSCs were normalized against the value obtained from Con to assess the relative telomerase activity (RTA).

\section{Western blotting}

TERT expression in Con and differentiated DP-MSCs was assessed in triplicates by western blotting. Protein extraction, quantification and fractionation (electrophoresis) were performed in accordance with previous article (Lee et al., 2019). The membrane was then blocked with $1 \%$ BSA for $1 \mathrm{~h}$, and was incubated with the primary antibodies in terms of anti-TERT (1:200 dilution; Santa Cruz Biotechnology, CA, USA) or anti-ACTB $(1: 1,000$; Cell Signaling, MA, USA) at $4^{\circ} \mathrm{C}$ for overnight. Thereafter, the membranes were incubated with proper horseradish peroxidase-conjugated secondary antibodies (1:3,000 dilution; Santa Cruz biotechnology) for $1 \mathrm{~h}$ at room temperature. The membranes were treated with enhanced chemiluminescence (Amersham Biosciences Corp, NJ, USA) and were exposed to X-ray film. Signals of TERT were scanned, were evaluated by Image J, and were normalized against intensity from ACTB.

\section{Statistical analysis}

The statistical significance was analyzed using one-way analysis of variance (ANOVA) using SPSS 12.0 (SPSS Inc. IL. USA) followed by Games-Howell post hoc analysis. All data were presented as mean \pm standard error (SEM). A value of $p<0.05$ was considered as statistically significant difference.

\section{RESULTS}

\section{Characterization of DP-MSCs}

Whereas MSCs-specific cell surface molecules (CD44, CD90 and CD105) were strongly positive, hematopoietic cell marker (CD45) was negative in DP-MSCs (Fig. 1A). In addition, intracellular lipid droplets in adipogenesis or calcium deposit in osteogenesis were gradually accumulated or identified in a time dependent manner, respectively (Fig. 1B). Therefore, it was confirmed that DP-MSCs in the present study were homogenous MSCs population, 

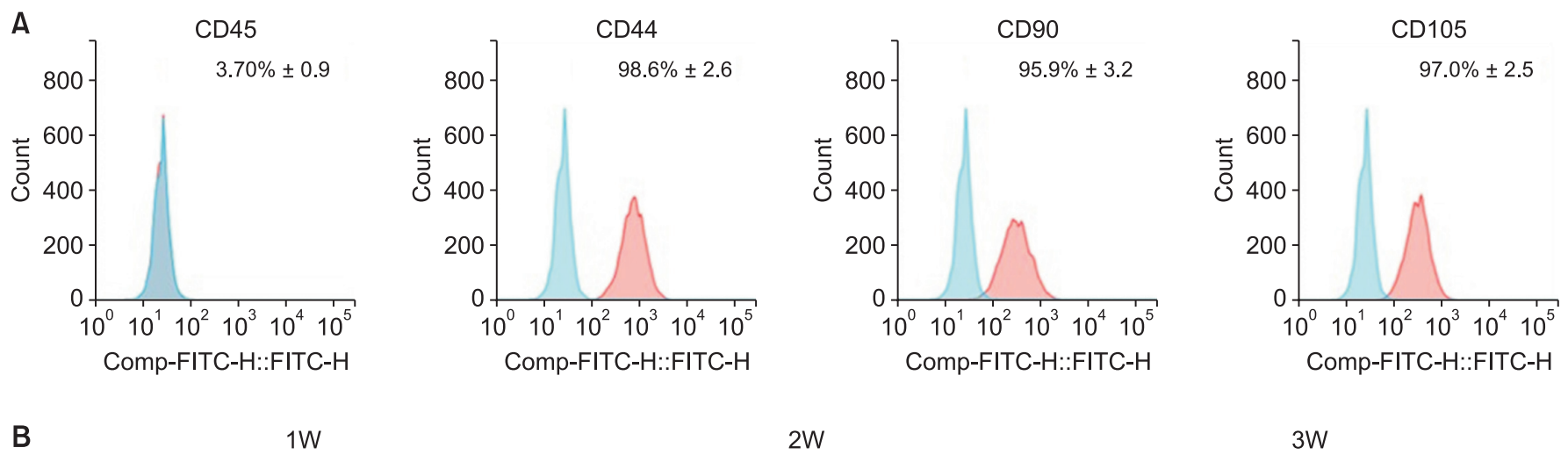

$2 \mathrm{~W}$

$3 \mathrm{~W}$

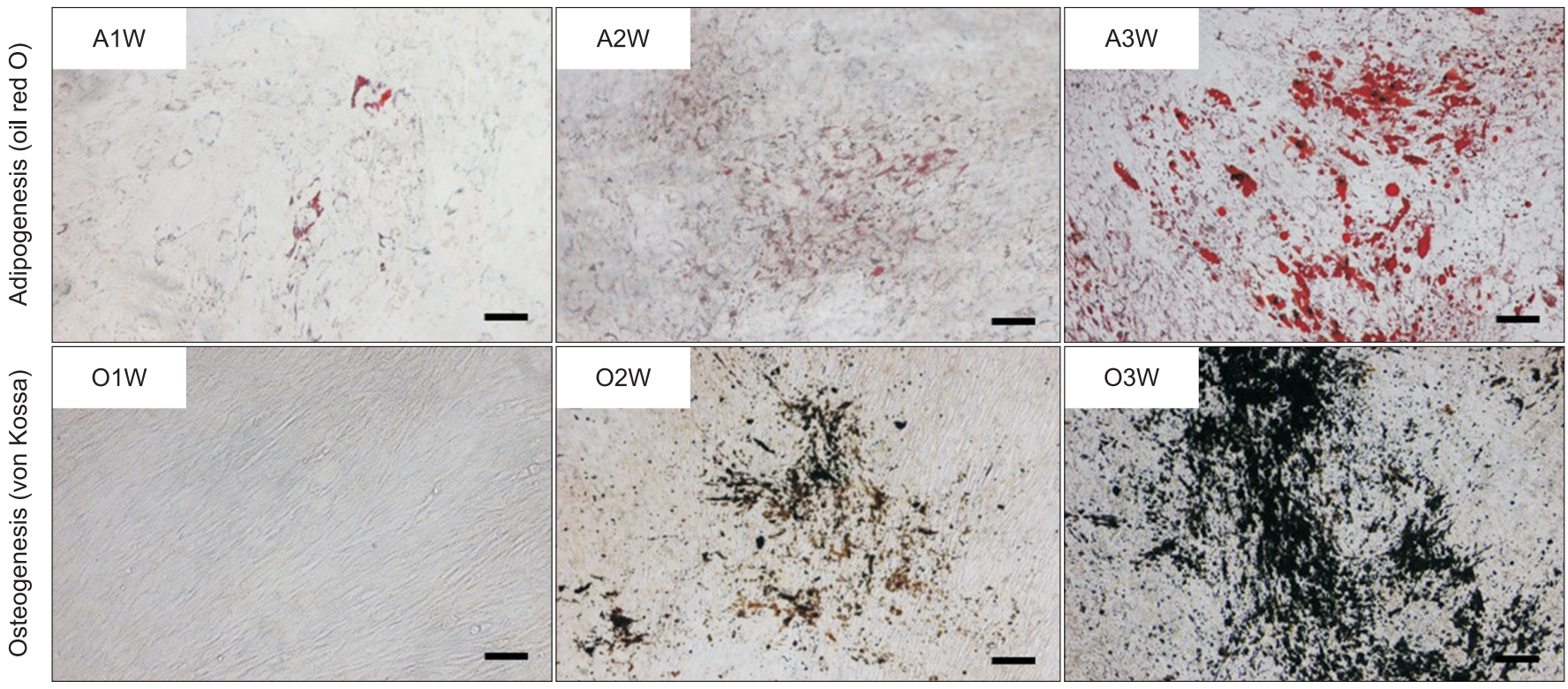

Fig. 1. Characterization of DP-MSCs. (A) Investigation for MSCs-specific surface molecule expression by flow cytometry. The ratios of positive cells were presented as mean\% \pm SEM. (B) Differentiation capacity of DP-MSCs into adipocytes (Oil red O staining) and osteoblasts (von Kossa staining) on a weekly basis up to 3 weeks. DP-MSCs, Dental pulp-derived mesenchymal stem cells; A1W, A2W and A3W, differentiated DP-MSCs for 1, 2 and 3 weeks into adipocytes; O1W, O2W and O3W, differentiated DP-MSCs for 1, 2 and 3 weeks into osteoblasts; Magnification: $\times 40$; Scale bar: $100 \mu \mathrm{m}$.

and were successfully differentiated for further analysis.

\section{Assessment of telomere length}

Telomere length in Con and differentiated DP-MSCs was evaluated using 2 different methods, southern blotting and qRT-PCR (Fig. 2 and 3). In non-radioactive chemiluminescent assay (southern blotting), the mean \pm SEM of the telomere length was $10.5 \pm 0.6,10.1 \pm 0.7,9.4 \pm 0.6$, $8.6 \pm 0.1,8.4 \pm 0.4,8.2 \pm 0.3$ and $8.1 \pm 0.3 \mathrm{~kb}$ in Con, $\mathrm{A} 1 \mathrm{~W}, \mathrm{~A} 2 \mathrm{~W}, \mathrm{~A} 3 \mathrm{~W}, \mathrm{O} 1 \mathrm{~W}, \mathrm{O} 2 \mathrm{~W}$ and $\mathrm{O} 3 \mathrm{~W}$, respectively (Fig. $2 \mathrm{~A})$. The telomere lengths at the late adipogenesis ( $\mathrm{A} 3 \mathrm{~W}$ ) and from the early osteogenesis (O1W-O3W) of DP-MSCs were significantly $(p<0.05)$ shorter than that of Con (Fig. $2 \mathrm{~B})$. In the relative telomere length assay using qRT-PCR, values from the middle stage of both adipogenic (A2W$\mathrm{A} 3 \mathrm{~W})$ and osteogenic (O2W-O3W) differentiation were significantly $(p<0.05)$ lower than those from Con and early stage (A1W and O1W) of differentiation (Fig. 2C). Of particular, once telomere length of MSCs was shortened under differentiation induction, it tended to remain shorter than undifferentiated state (Con).

\section{Assessment of telomerase activity}

The RTA in Con and differentiated DP-MSCs was determined by RQ-TRAP. The value in Con was considered as 1 for comparison with other groups. Levels of RTA showed significantly $(p<0.05)$ extensive decrease since the early stages of both differentiation (A1W and O1W) than Con, 

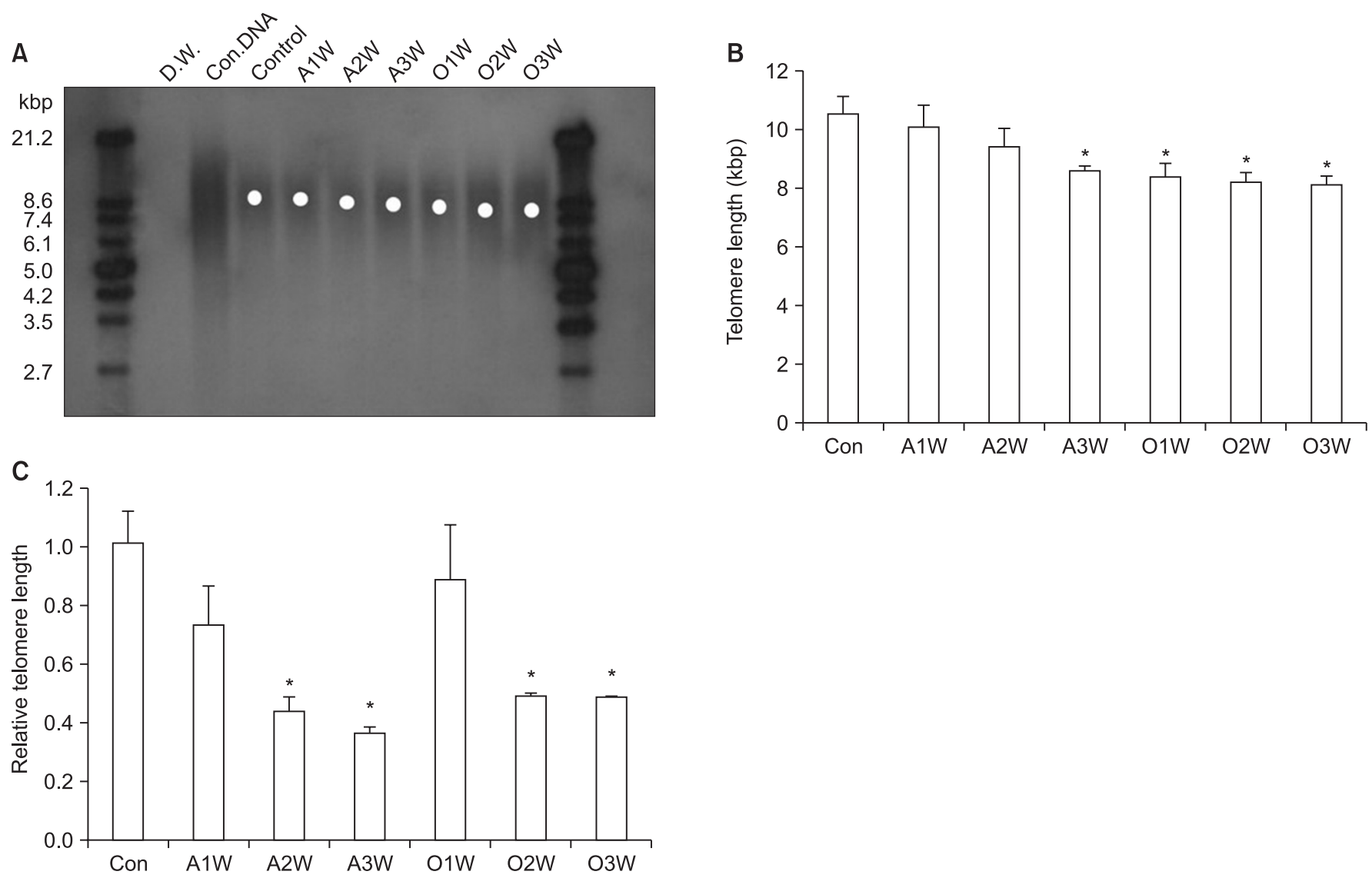

Fig. 2. Assessment of the telomere length by southern blotting and qRT-PCR. (A) The representative image from non-radioactive chemiluminescent assay (southern blotting). White dots indicated the mean value of telomere restriction fragment lengths (TRF). (B) Mean of TRF in Con and differentiated DP-MSCs. (C) Telomere length in Con and differentiated DP-MSCs by qRT-PCR. Asterisks in the top of bars indicated significant $(p<0.05)$ differences, in comparison with Con. Graphs were presented as mean \pm SEM. D.W., distilled water; Con. DNA, control DNA.

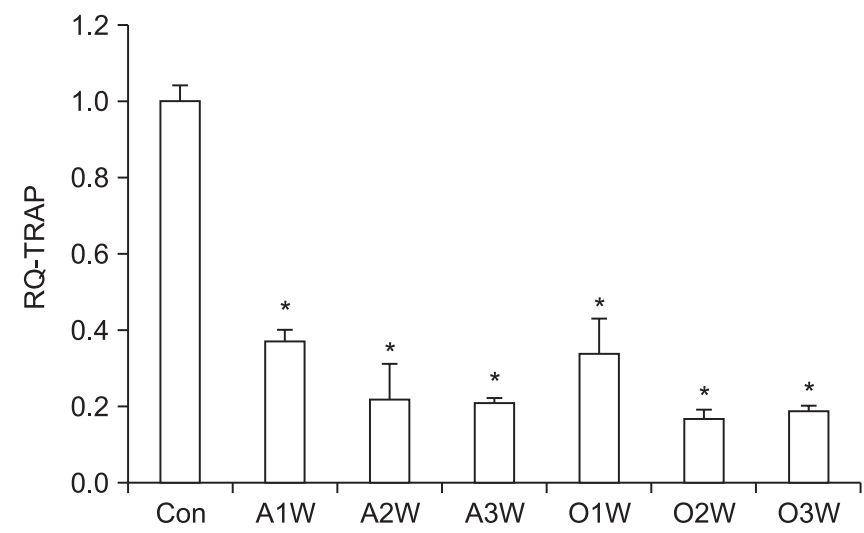

Fig. 3. Relative telomerase activity (RTA) by relative-quantitative telomerase repeat amplification protocol (RQ-TRAP) assay in Con and differentiated DP-MSCs. Asterisks in the top of bars indicated significant $(p<0.05)$ differences, in comparison with Con. Graphs were presented as mean \pm SEM. and remained lower levels along with prolonged differentiation durations (Fig. 3).

\section{Investigation of TERT expression}

The expression of TERT was examined in Con and differentiated DP-MSCs, and the bands were normalized against the intensity from ACTB (Fig. 4A). Whereas TERT expressions in the early stages of both differentiations (A1W and O1W) were similar with that of Con, the levels were significantly $(p<0.05)$ decreased from the middle stages of adipogenic (A2W-A3W) and osteogenic (O2WO3W) differentiation inductions in DP-MSCs (Fig. 4B). Of particular, TERT expression was almost undetectable from the middle stage $(\mathrm{O} 2 \mathrm{~W}-\mathrm{O} 3 \mathrm{~W})$ of osteogenesis. 
A

$$
\text { TERT (120 kda) }
$$

ACTB (43 kda)

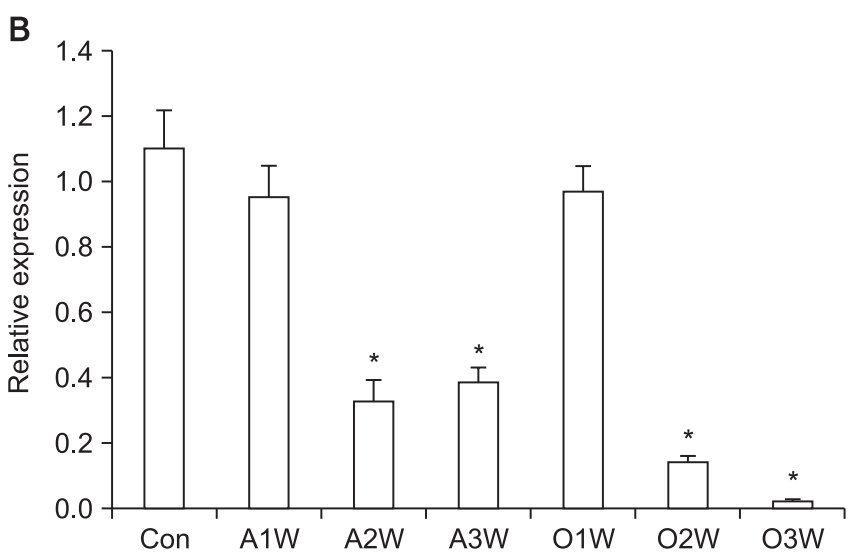

Fig. 4. Changes of protein levels in TERT expression. (A) Representative image from western blotting. Lanes, which were displayed from the left to the right, showed Con, A1W, A2W, A3W, O1W, O2W and O3W. (B) The relative intensities of TERT expression after normalization against ACTB. Asterisks in the top of bars indicated significant $(p<0.05)$ differences, in comparison with Con. Graphs were presented as mean \pm SEM.

\section{DISCUSSION}

The present study investigated the periodical alterations of telomere length, telomerase activity and TERT expression in differentiated MSCs. Overall, differentiation induction shortened telomere length and weakened telomerase activity with TERT expression from early-middle stage of differentiation. Of particular, telomere length shortening was remained along with prolonged differentiation durations, possibly due to weakened telomerase activity and TERT expression.

In the previous results, it has been well addressed that telomere length in MSCs is longer than adult somatic cells. Longer lengths have been reported in stem cells (10$15 \mathrm{~kb})$ in comparison with somatic cells $(5-15 \mathrm{~kb}$ ) (Hiyama and Hiyama 2007). When DP-MSCs, UCM-MSCs, AT-MSCs and somatic cells (fibroblasts and adult muscle cells) were comparatively investigated, telomere length was significantly $(p<0.05)$ higher in MSCs group $(\sim \mathrm{kb})$ than somatic cells $(\sim 7 \mathrm{~kb})$ (Jeon et al., 2011b). In addition, mean of telomere lengths in several types of MSCs was $11 \mathrm{~kb}$, but fibroblast exhibited $7 \mathrm{~kb}$ (Jeon et al., 2011a). The telomere length in DP-MSCs from adult chimpanzee has been demonstrated as more than $10 \mathrm{~kb}$ (Cheng et al., 2008).
In agreement these results, the telomere length in Con was $10.5 \mathrm{~kb}$, and the late stage of differentiated DP-MSCs which can be regarded as the adult somatic cell exhibited shorter telomere length as $8.6 \mathrm{~kb}$ in adipogenesis (A3W) and $8.1 \mathrm{~kb}$ in osteogenesis (O3W) (Fig. 2). Therefore, it indicated that telomere length in differentiated MSCs could be shortened to the same level of adult somatic cell.

Telomerase activity has been known for up-regulation in stem cells because it plays an essential role in maintenance of undifferentiated status, self-renewal and differentiation ability into a various cell lineages (Armstrong et al., 2000). MSCs with lacking telomerase showed decreases of pluripotency and differentiation capacity (Liu et al., 2004). Furthermore, differentiated immortal cells including cancer cell and ESCs showed decreased telomerase activity compared to undifferentiated status (Sharma et al., 1995). In the present study, differentiated DP-MSCs also showed drastic decrease of telomerase activity even within the early stage of differentiation induction (A1W and $\mathrm{O} 1 \mathrm{~W}$ ), and remained lower levels along with prolonged differentiation durations (Fig. 3). This observation could be supported by several reports which demonstrate that MSCs revealed higher level of telomerase activity than somatic cells. Levels of telomerase activity in DPMSCs, UCM-MSCs and AT-MSCs were higher than those of fibroblasts and somatic cells (Jeon et al., 2011a; Jeon et al., 2011b). When several passaged (1, 5, 10, 15, and 20 passages) MSCs and fibroblasts were comparatively investigated, the level of telomerase activity was higher in porcine BM-MSCs than fibroblasts at all the passages (Jeon et al., 2011c).

Stable preservation or elongation of telomere length is highly related with telomerase activity. The telomere length in immortal cell lines in terms of cancer cell and ESCs can be maintained by activation of telomerase (Chan and Blackburn, 2004). On the other hand, it has been addressed that telomerase activity is undetectable in the normal somatic cells, and is identified as low to moderate level in case of MSCs (Harle-Bachor and Boukamp, 1996). In agreement with this observation, the late stage of both differentiations ( $\mathrm{A} 3 \mathrm{~W}$ and $\mathrm{O} 3 \mathrm{~W}$ ) which can be considered as the adult somatic cell presented extremely low level of RTA (0.2-fold) in comparison with Con (Fig. 3). TERT is known as the telomerase catalytic gene, an indicator of telomerase activity, and mainly regulates the telomerase activity (Meyerson et al., 1997). As shown in Fig. 2, both 
telomere length assays demonstrated that telomere shortening was remained along with prolonged differentiation durations (Fig. 2). It could be explained that weakened telomerase activity and TERT expression during differentiation of DP-MSCs resulted in failure to preserve or elongate telomere length (Fig. 3 and 4).

In conclusion, the present study demonstrated that differentiation induction in MSCs could affect to telomere length, telomerase activity and TERT expression. We suggest that understanding of cellular alterations during differentiation of MSCs is a vital step in order to ensure safety in the transplantable cell application and standardize therapeutic strategies in the field of biotechnology.

\section{CONFLICTS OF INTEREST}

No potential conflict of interest relevant to this article was reported.

\section{ACKNOWLEDGEMENTS}

This work was supported by a grant from the National Research Foundation (NRF) of Korea, funded by the government of the Republic of Korea (grant no. NRF2017R1C1B5076029).

\section{ORCID}

Hyeon-Jeong Lee: https://orcid.org/0000-0002-2154-239X Ryoung-Hoon Jeon: https://orcid.org/0000-0003-3174-1197 Byung-Joon Park: https://orcid.org/0000-0003-1901-0869 Si-Jung Jang: https://orcid.org/0000-0003-3829-6807 Sung-Lim Lee: https://orcid.org/0000-0002-1055-8097 Gyu-Jin Rho: https://orcid.org/0000-0002-6264-0017 Seung-Joon Kim: https://orcid.org/0000-0002-8521-8898 Won-Jae Lee: https://orcid.org/0000-0003-1462-7798

\section{REFERENCES}

Armstrong L, Lako M, Lincoln J, Cairns PM, Hole N. 2000. mTert expression correlates with telomerase activity during the differentiation of murine embryonic stem cells. Mech. Dev. 97:109-116.

Artandi SE, DePinho RA. 2010. Telomeres and telomerase in cancer. Carcinogenesis 31:9-18.

Campisi J, Yaswen P. 2009. Aging and cancer cell biology. Aging Cell 8:221-225.
Chan SR, Blackburn EH. 2004. Telomeres and telomerase. Philos. Trans. R. Soc. Lond. B. Biol. Sci. 359:109-122.

Cheng PH, Snyder B, Fillos D, Ibegbu CC, Huang AHC, Chan AWS. 2008. Postnatal stem/progenitor cells derived from the dental pulp of adult chimpanzee. BMC Cell Biol. 9:20.

Gonzalo S, Jaco I, Fraga MF, Chen T, Li E, Esteller M, Blasco MA. 2006. DNA methyltransferases control telomere length and telomere recombination in mammalian cells. Nat. Cell Biol. 8:416-424.

Harle-Bachor C, Boukamp P. 1996. Telomerase activity in the regenerative basal layer of epidermis in human skin and in immortal and carcinoma-derived skin keratinocytes. Proc. Natl. Acad. Sci. 93:6476-6481.

Hiyama E, Hiyama K. 2007. Telomere and telomerase in stem cells. Br. J. Cancer 96:1020-1024.

Jeon BG, Kang EJ, Kumar BM, Maeng GH, Ock SA, Kwack DO, Park BW, Rho GJ. 2011a. Comparative Analysis of Telomere Length, Telomerase and Reverse Transcriptase Activity in Human Dental Stem Cells. Cell Transplantation 20:16931705.

Jeon BG, Kumar BM, Kang EJ, Ock SA, Lee SL, Kwack DO, Byun JH, Park BW, Rho GJ. 2011b. Characterization and comparison of telomere length, telomerase and reverse transcriptase activity and gene expression in human mesenchymal stem cells and cancer cells of various origins. Cell Tissue Res. 345:149-161.

Jeon BG, Kwack DO, Rho GJ. 2011c. Variation of Telomerase Activity and Morphology in Porcine Mesenchymal Stem Cells and Fibroblasts during Prolonged in vitro Culture. Animal Biotechnology 22:197-210.

Lee HJ, Park BJ, Jeon RH, Jang SJ, Son YB, Lee SL, Rho GJ, Kim SJ, Lee WJ. 2019. Alteration of Apoptosis during Differentiation in Human Dental Pulp-Derived Mesenchymal Stem Cell. J. Anim. Reprod. Biotechnol. 34:2-9.

Liu L, Di Girolamo CM, Navarro PA, Blasco MA, Keefe DL. 2004. Telomerase deficiency impairs differentiation of mesenchymal stem cells. Exp. Cell Res. 294:1-8.

Meyerson M, Counter CM, Eaton EN, Ellisen LW, Steiner P, Caddle SD, Ziaugra L, Beijersbergen RL, Davidoff MJ, Liu Q, Bacchetti S, Haber DA, Weinberg RA. 1997. hEST2, the putative human telomerase catalytic subunit gene, is up-regulated in tumor cells and during immortalization. Cell 90:785-795.

Parsch D, Fellenberg J, Brümmendorf TH, Eschlbeck AM, Richter W. 2004. Telomere length and telomerase activity during expansion and differentiation of human mesenchymal stem cells and chondrocytes. J. Mol. Med. (Berl.) 82:49-55.

Sharma HW, Sokoloski JA, Perez JR, Maltese JY, Sartorelli AC, Stein CA, Nichols G, Khaled Z, Telang NT, Narayanan R. 1995. Differentiation of immortal cells inhibits telomerase activity. Proc. Natl. Acad. Sci. USA 92:12343-12346. 